\title{
Teaching Video NeuroImages: Acute hemichorea-hemiballism reverted after IV thrombolysis
}

Caio Disserol, MD, Bárbara Alencar, MD, Jacy Parmera, MD, Adriana Bastos Conforto, MD, PhD, and Lécio Figueira Pinto, MD, PhD

Neurology ${ }^{\circledR}$ 2020;94:e121-e122. doi:10.1212/WNL.0000000000008706

Figure Imaging

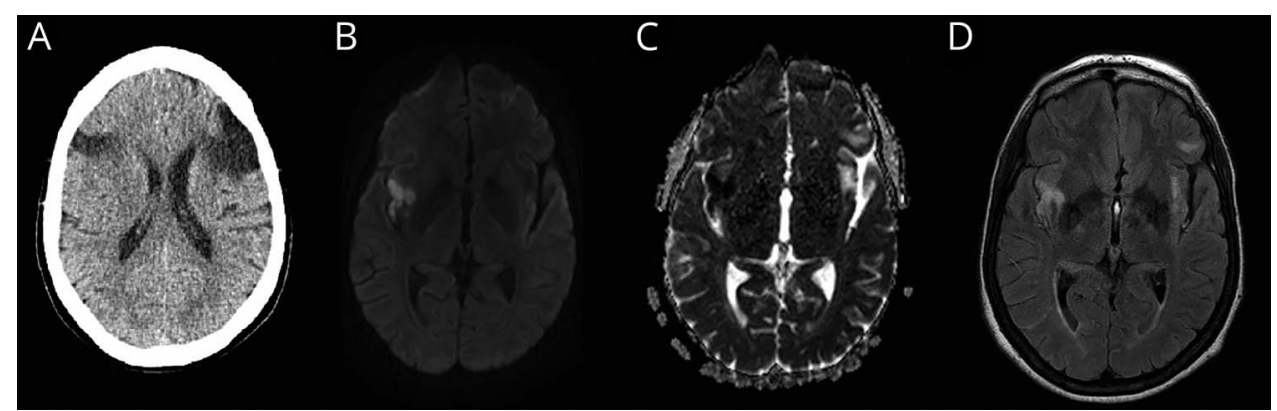

Head CT scan shows old infarcts (A) and brain MRI shows an acute stroke involving the right insular cortex and capsula extrema on diffusion-weighted (B), apparent diffusion coefficient map (C), and fluid-attenuated inversion recovery (D) images.

A 59-year-old woman was admitted with acute left hemichorea-hemiballism. Blood glucose level was $87 \mathrm{mg} / \mathrm{dL}$. Head CT scan showed old infarcts (figure). The patient underwent thrombolysis with IV alteplase $(0.9 \mathrm{mg} / \mathrm{kg})$ within 86 minutes of symptom onset, evolving with partial improvement after 2 hours (video) and complete resolution after 24 hours without other treatments. Brain MRI showed an acute stroke in the right insula (figure), known to be functionally connected to the posterolateral putamen. ${ }^{1}$ Hemichorea-hemiballism is an uncommon presentation of stroke and may be caused by insular, putaminal, and various other lesions connected to the same network. ${ }^{1,2}$

\section{Study funding}

No targeted funding reported.

\section{Disclosure}

The authors report no disclosures relevant to the manuscript. Go to Neurology.org/N for full disclosures.

\section{References}

1. Laganier S, Boes A, Fox M. Network localization of hemichorea-hemiballismus. Neurology 2016;86:2187-2195.

2. Bembenek JP, Bilik M, Członkowska A. Successful treatment with intravenous recombinant tissue plasminogen activator in an acute stroke patient presenting with hemiballism. Funct Neurol 2015;30:71-72.

\section{Correspondence}

Dr. Disserol

caio.disserol@hc.fm.usp.br

\section{MORE ONLINE}

\section{- Video}

$\rightarrow$ Teaching slides

links.lww.com/WNL/

B19 
Appendix Authors

\begin{tabular}{|c|c|c|c|}
\hline Name & Location & Role & Contribution \\
\hline $\begin{array}{l}\text { Caio } \\
\text { Disserol, } \\
\text { MD }\end{array}$ & $\begin{array}{l}\text { Hospital das } \\
\text { Clínicas, University } \\
\text { of São Paulo } \\
\text { Medical School }\end{array}$ & Author & $\begin{array}{l}\text { Wrote the manuscript, } \\
\text { data collection, analysis, } \\
\text { and interpretation }\end{array}$ \\
\hline $\begin{array}{l}\text { Bárbara } \\
\text { Alencar, } \\
\text { MD }\end{array}$ & $\begin{array}{l}\text { Hospital das } \\
\text { Clínicas, University } \\
\text { of São Paulo } \\
\text { Medical School }\end{array}$ & Author & $\begin{array}{l}\text { Data collection, analysis, } \\
\text { and interpretation }\end{array}$ \\
\hline $\begin{array}{l}\text { Jacy } \\
\text { Parmera, } \\
\text { MD }\end{array}$ & $\begin{array}{l}\text { Hospital das } \\
\text { Clínicas, University } \\
\text { of São Paulo } \\
\text { Medical School }\end{array}$ & Author & $\begin{array}{l}\text { Conceptualization of the } \\
\text { study, interpretation of } \\
\text { the data, revision of the } \\
\text { manuscript }\end{array}$ \\
\hline $\begin{array}{l}\text { Adriana } \\
\text { Bastos } \\
\text { Conforto, } \\
\text { MD, PhD }\end{array}$ & $\begin{array}{l}\text { Hospital das } \\
\text { Clínicas, University } \\
\text { of São Paulo } \\
\text { Medical School }\end{array}$ & Author & $\begin{array}{l}\text { Interpretation of the } \\
\text { data, revision of the } \\
\text { manuscript }\end{array}$ \\
\hline $\begin{array}{l}\text { Lécio } \\
\text { Figueira } \\
\text { Pinto, MD, } \\
\text { PhD }\end{array}$ & $\begin{array}{l}\text { Hospital das } \\
\text { Clínicas, University } \\
\text { of São Paulo } \\
\text { Medical School }\end{array}$ & Author & $\begin{array}{l}\text { Conceptualization of the } \\
\text { study, interpretation of } \\
\text { the data, revision of the } \\
\text { manuscript }\end{array}$ \\
\hline
\end{tabular}




\section{Neurology}

\section{Teaching Video NeuroImages: Acute hemichorea-hemiballism reverted after IV thrombolysis}

Caio Disserol, Bárbara Alencar, Jacy Parmera, et al. Neurology 2020;94; $121-\mathrm{e} 122$

DOI 10.1212/WNL.0000000000008706

This information is current as of December 30, 2019

\section{Updated Information \&} Services

References

Subspecialty Collections

Permissions \& Licensing

Reprints including high resolution figures, can be found at: http://n.neurology.org/content/94/1/e121.full

This article cites 2 articles, 1 of which you can access for free at: http://n.neurology.org/content/94/1/e121.full\#ref-list-1

This article, along with others on similar topics, appears in the following collection(s):

\section{All Cerebrovascular disease/Stroke}

http://n.neurology.org/cgi/collection/all_cerebrovascular_disease_strok e

\section{Chorea}

http://n.neurology.org/cgi/collection/chorea

MRI

http://n.neurology.org/cgi/collection/mri

Information about reproducing this article in parts (figures,tables) or in its entirety can be found online at:

http://www.neurology.org/about/about_the_journal\#permissions

Information about ordering reprints can be found online:

http://n.neurology.org/subscribers/advertise

Neurology ${ }^{\circledR}$ is the official journal of the American Academy of Neurology. Published continuously since 1951, it is now a weekly with 48 issues per year. Copyright @ 2019 American Academy of Neurology. All rights reserved. Print ISSN: 0028-3878. Online ISSN: 1526-632X.

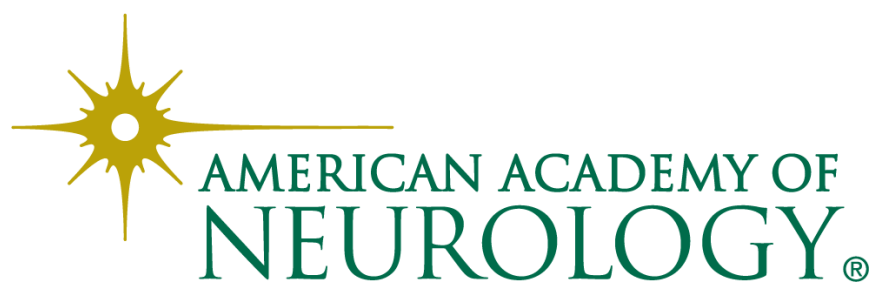

